culture. Conclusions: Based on the results, our recommendations for increasing TRIP are: (1) ensure integrated development of a master data warehouse; (2) improve communication with special emphasis on involving researchers more directly with DS teams; (3) promote further research to identify what works/does not work in implementing TRIP; and (4) 'think globally, but translate locally.' Funded by NIH/AHRQ for the HMORN CCSN (Coordinated Clinical Studies Network).

\section{Abstract PS1-41 \\ A Diabetes Quality Improvement Project at a Rural Primary Care Clinic}

Gail A. Bloch, RN, BSBA, Marshfield Clinic Merrill Center; Trina J. Ford, MSN, RD, Marshfield Clinic; Marilyn Hodgson, RN, CDE, Marshfield Clinic Merrill Center; Paula McIntyre, CDE, RD, Marshfield Clinic Merrill Center; Michael Sheehan, MD, Marshfield Clinic Weston Center; Kathryn Krohn-Gill, MD, Marshfield Clinic Merrill Center; Kelley Natzke, RN, Marshfield Clinic Merrill Center; Eric Penniman, DO, Marshfield Clinic Stettin Center

Background: In reviewing first quarter clinical quality metrics in 2006, the primary care department at the Merrill Center identified a statistically significant drop in glycemic control for their diabetic population that did not occur across the clinic system-wide. Quality improvement changes were implemented from mid 2006 through mid 2007. Aim: To increase glycemic control more than $50 \%$ over 12 months as evidenced by the percentage of patients with diabetes having most recent A1c test results $<7 \%$. Methods: The system-wide quality improvement initiative increased provider awareness and educated providers on the need to improve clinical outcomes for patients with diabetes. Providers partnering with nurse educators resulted in increased referrals to the Diabetes Self Management program. Diabetes educators intensified insulin dosing for patients with uncontrolled blood sugars through use of a standard protocol. In addition to expanding existing diabetes services and hours, telephonic care management was initiated for high risk patients. Implementation: An electronic medical record (EMR) and tablet computers improved the information that providers and other members of the care team could use to access at the point of service. An electronic reminder system helps identify patients not at goal requiring further testing, education, or medical intervention. A process for gathering outside A1c test results improved the coordination of diabetes care across health care facilities. Results: Glycemic control as evidenced by the percentage of patients with most recent A1c test results $<7 \%$ rose from $41 \%$ in second quarter of 2006 to $68.2 \%$ in third quarter of 2007 for the more than 800 patients with diabetes. Conclusions: To improve quality of care, provider awareness and support are essential in developing a comprehensive diabetes program. Increased provider awareness, supported by an EMR has built a team approach to education, medical management, and care coordination resulting in improved glycemic control.

Abstract PS1-42

\section{Demonstrating the Effective Practice}

James W. Dearing, PhD, Kaiser Permanente Colorado; R. Sam Larson, PhD, Kaiser Permanente Colorado

Background: For 160 years, government agencies, corporations, and nonprofit organizations have relied on demonstrations of radical innovations and interventions to stimulate interest in them and subsequent diffusion. Unfortunately, researchers and practitioners alike often fail to distinguish the functions that a demonstration project may serve and, thus, put into practice hybrid demonstration forms that typically under-achieve in all functional objectives. Methods: We (1) visited internal demonstrations of effective practices in primary care, palliative care, and population preventive services, (2) discussed the planning of demonstrations with national strategic support staff, and (3) reviewed the published literature about demonstration projects. Results: In Kaiser Permanente nationally, demonstration projects are often initiated without regard to functional specification, perceptions of potential adopters, communication training of demonstration staff, or knowledge of published literature about the operation of demonstration projects. Conclusions: The potential for improving the internal demonstration of effective practices in integrated healthcare delivery systems is large. Cost-effective strategies exist to improve the decision making of potential adopters, as well as accelerate the dissemination of effective practices through formative evaluation about the credibility of potential adopters, potential adopters' perceptions of practices to be demonstrated, staff training, and implementation support.

\section{Abstract PS1-44 \\ Using Fault Tree Analysis and Probabilistic Risk Assessment to Improve Medication Safety in Ambulatory Care}

Terry S. Field, DSc, Meyers Primary Care Institute; Larry Garber, MD, Fallon Clinic; Kevin Chysna, BA, Meyers Primary Care Institute; Brooke Harrow, PhD, Fallon Clinic; Jennifer Tjia, MD, MPH, Meyers Primary Care Institute; Jerry H. Gurwitz, MD, Meyers Primary Care Institute

Background: In a previous study of adverse drug events (ADEs) in the ambulatory setting, we found that $42 \%$ of the serious, life-threatening, and fatal ADEs were preventable. We identified 7 types of errors in medication prescribing and monitoring that were the proximal causes of these events: 1) prescribing a medication for which the patient has a history of allergy, 2) a medication that conflicts with recent laboratory values or the patient's medical conditions, 3) a medication that interacts with other medications the patient is taking or 4) an excessive dose, 5) inadequate monitoring or 6) not acting on the results of monitoring, or 7) the patient does not use prophylaxis for known side effects of the medication. The aim of this study is to assess the feasibility of applying fault tree analysis and probabilistic risk assessment to the analysis of errors contributing to ADEs. Methods: We applied probabilistic risk assessment techniques borrowed from the safety engineering industry to identify system-level interventions for reducing the rate of ADEs. Interdisciplinary clinical including primary care and specialist physicians, advanced practice nurses, nurse managers, and pharmacists were convened to construct fault trees (graphic representations of events contributing to an undesired outcome) for the 7 types of errors in prescribing and monitoring medications. We are using probabilistic risk assessment software (SAPHIRE) to evaluate the fault trees, develop cut-sets, and identify and analyze potential system-level interventions to prevent ADEs. Results: Participants learned to develop complex and thorough fault trees with little difficulty. The clinicians quickly became comfortable detailing the systemic and individual processes that lay behind serious medication errors. The fault trees reveal an array of opportunities to implement interventions with a high probability of improving patient safety. Conclusions: Techniques for risk reduction from industry appear to be of value in assessing and improving safety problems related to medication prescribing and monitoring.

\section{Abstract PS1-45 \\ The Johns Hopkins Hospital at Home Care for Acutely Ill Older Persons: A Model for Managed Care}

Bruce Leff, MD, Johns Hopkins University School of Medicine; Lynda Burton, ScD, Johns Hopkins University Bloomberg School of Public Health; Susan Guido, RN, Johns Hopkins Bayview Medical Center; Scott Mader, MD, Portland Veterans Administration Medical Center Oregon Health and Science University; Bruce Naughton, MD, State University of New York; Kevin Frick, PhD, Johns Hopkins University Bloomberg School of Public Health; Jeffrey Burl, MD, Fallon Community Health Plan and Fallon Clinic; John Burton, MD, Johns Hopkins University School of Medicine; Donald Steinwachs, PhD, Johns Hopkins University Bloomberg School of Public Health

Background: Acute hospital care of older patients is often associated with adverse events including functional decline, iatrogenic illness, dissatisfaction with care among patients and family members, family stress related to caring for a family member, and high costs. Delivering acute medical care at home in a hospital at home $(\mathrm{HaH})$ that substitutes for traditional acute hospital care may be associated with better outcomes in these domains. Aim: Describe results of a national demonstration study of a $\mathrm{HaH}$ model of care in Medicare managed care and Veterans Administration (VA) settings. Methods: Prospective, nonrandomized clinical trial, in which acutely ill older persons (age $>65$ ) who met required hospital level care for community-acquired pneumonia, congestive heart failure, chronic obstructive pulmonary disease, and cellulitis were followed through their usual hospital course during an observation phase $(\mathrm{n}=286)$ and were offered $\mathrm{HaH}$ care during an intervention phase $(\mathrm{n}=141)$. The study populations were from three Medicare managed 